\title{
РАЗРАБОТКА ИНСТРУМЕНТАРИЯ ИССЛЕДОВАНИЯ ГРУППОВОГО И МИКРОГРУППОВОГО КОНФЛИКТА В ПРОИЗВОДСТВЕННЫХ ГРУППАХ
}

Андрей Владимирович Сидоренков

доктор психологических наук, профессор

Южный федеральный университет

2. Ростов-на-Дону, Россия

E-mail: sav@micro-cosm.ru

Олеся Юрьевна Шипитько

кандидат психологических наук, преподаватель

Южный федеральный университет

2. Ростов-на-Дону, Россия

E-mail: lavnes@mail.ru

Наталья Юрьевна Ульянова

кандидат психологических наук, преподаватель

Южный федеральный университет

2. Ростов-на-Дону, Россия

E-mail:n.y.ulyanova@gmail.com

\section{Сергей Васильевич Сарычев}

доктор психологических наук, профессор Курский государственный университет 2. Курск, Россия

E-mail: sarychev@r46.ru

Статья подготовлена при финансовой поддержке РГНФ в рамках научноисследовательского проекта «Противоречия, конфликты и эффективность малых групп в организачиях и на предприятиях», № 16-36-00006

В статье рассмотрены существующие методы и методики, прежде всего опросного типа, для изучения конфликтов в малых производственных группах. Приведены данные о методиках изучения конфликта и особенности их применения. Представлена концептуальная платформа, содержащая понимание природы конфликта и модель его проявления в малой группе, которая легла в основу создания опросника. 
Описан разработанный опросник типов группового и микрогруппового конфликта. Он состоит из двух частей для изучений уровней группового и микрогруппового конфликта и имеет две субшкалы для измерения двух типов конфликта (деятельностно-ориентированного и субъектно-ориентированного) на каждом из этих уровней. Опросник содержит восемь айтемов, по четыре в каждой субшкале. Оченка по айтемам осуществляется членами группы по семибалльной шкале типа шкалы Лайкерта. На выборке 18 малых производственных групn ( $N=200$ работников), представляющих собой первичные структурные подразделения в организациях, было проведено исследование. Была сделана экспертиза с привлечением трех специалистов-психологов и 25 работников из производственных групn, отобранных случайным образом. Проведена прочедура оченки содержательной, очевидной и дискриминантной валидности, надежности-согласованности субшкал опросника, а также эмпирического распределения. Доказана правомерность выделения в представленном опроснике двух субшкал. Показано, что он обладает валидностью и надежностью по всем проанализированным показателям. Опросник может применяться как для решения научно-исследовательских, так и практических задач. В статье сделаны основные выводы, освещены возможности использования опросника типов группового и микрогруппового конфликта.

Ключевые слова: диагностика конфликта, опросник, типы конфликта, уровни конфликта, групповой конфликт, микрогрупповой конфликт, деятельностно-ориентированный конфликт, субъектно-ориентированный конфликт, малая группа, производственные группы.

Для цитирования: Сидоренков А. В., Шипитько О. Ю., Ульянова Н. Ю., Сарычев С. В. Разработка инструментария исследования группового и микрогруппового конфликта в производственных группах // Российский психологический журнал. - 2016. - Т. 13. - № 3. - С. 89-106.

\title{
THE DEVELOPMENT OF TOOLS FOR STUDYING GROUP AND MICROGROUP CONFLICTS IN GROUPS OF EMPLOYEES
}

\author{
Andrei Vladimirovich Sidorenkov \\ Doctor of Psychology, Professor \\ Southern Federal University \\ Rostov-on-Don, Russia \\ E-mail:sav@micro-cosm.ru
}


Olesia Iur'evna Shipit'ko

Candidate of Psychology, Lecturer

Southern Federal University

Rostov-on-Don, Russia

E-mail: lavnes@mail.ru

Natal'ia lur'evna Ul'ianova

Candidate of Psychology, Lecturer

Southern Federal University

Rostov-on-Don, Russia

E-mail: n.y.ulyanova@gmail.com

Sergei Vasil'evich Sarychev

Doctor of Psychology, Professor

Kursk State University

Kursk, Russia

E-mail: sarychev@r46.ru

\section{Acknowledgments}

This study was supported by the Russian Humanitarian Science Foundation grant "Contradictions, conflicts and effectiveness of small groups in organizations and enterprises" (research project no. 16-36-00006)

The paper analyses the existing methods and techniques (questionnaires, basically) for the study of conflict in small groups of employees. The authors provide the data on methods of studying conflicts and features of their application. A conceptual platform, containing understanding of the nature of conflict and model of its manifestation in small groups, formed the basis for the development of an inventory.

The paper describes the developed inventory for the study of types of group and microgroup conflicts. The inventory consisted of two parts for studying the levels of group and microgroup conflicts, and also had two subscales for measuring two types of conflict (activity-oriented and subject-oriented) on each of these levels. The inventory contained eight items (four in each subscale). The members of the group evaluated items on a seven-point Likert-type scale. The study involved 18 small groups of employees ( $N=200$ employees) that represented the primary structural units in organizations. The examination involved three psychologists and 25 persons from groups of employees selected at random. The authors evaluated (a) construct, convergent, and discriminant validity, (b) reliability-consistency of the subscales of the inventory, and (c) the empirical distribution. The study proved the appropriateness of distinguishing two subscales in the presented inventory. The inventory was valid 
and reliable for all the analyzed characteristics. The developed inventory can be readily used in practice, as well as in research work. The paper presents the main findings and suggests possible applications of the inventory for the study of types of group and microgroup conflicts.

Keywords: conflict diagnostics, inventory, types of conflict, levels of conflict, group conflict, microgroup conflict, activity-oriented conflict, subject-oriented conflict, small group, groups of employees.

For citation: Sidorenkov A. V., Shipit'ko O. Iu., Ul'ianova N. lu., Sarychev S. V. The Development of Tools for Studying Group and Microgroup Conflicts in Groups of Employees. Russian Psychological Journal, 2016, V. 13, no. 3, pp. 89-106.

\section{Введение}

Конфликт является неотъемлемой частью функционирования и развития производственных групп и команд. Он может оказывать позитивное или негативное действие на возникающие в группе социально-психологические явления (например, сплоченность, доверие, групповую действенность) и ее эффективность (продуктивность, качество работы, идей и инноваций, удовлетворенность членами группой и результатами ее деятельности). В связи с важной ролью, которую конфликты играют в жизнедеятельности групп, необходим инструментарий для изучения этого феномена в научно-исследовательских и практических целях. В настоящее время созданы методы, основанные на наблюдении, игровом и математическом моделировании, а также методики опросного типа для исследования тех или иных аспектов проявления конфликта.

Так, для анализа стратегий взаимодействия членов группы в конфликте применяют Систему многоуровневого наблюдения груnn (SYMLOG) (R. F. Bales \& S. P. Cohen, 1979) [8] и ее модификации (U. Becker-Beck, 1997) [11], для изучения поведения членов в конфликте в процессе решения проблемыКодирующую систему отношений в групповой работе (GWRCS) (M. S. Poole, 1983) $[14,21,24]$. Обе технологии основаны на методе наблюдения. С целью моделирования и анализа принятия решений в конфликте используется Графическая модель урегулирования конфликта (L. Fang et al., 1993) [13], расчеты и представление результатов которой обеспечены специально созданной программой Система поддержки принятия решений (GMCR I u GMCR II) (K. W. Hipel et al., 1999; D. M. Kilgour et al., 1996) [16, 20]. Также, в рамках экспериментального исследования конфликта в диадах и более многочисленных группах, широко используются игры, которые выполняют следующие функции: а) аналогия, модель фактических конфликтных ситуаций; б) эвристический элемент для обеспечения новых способов анализа 
конфликта; в) способ для отделения рациональных решений в конфликте от влияющих социальных и психологических факторов; г) простой экспериментальный инструмент для проверки теоретических гипотез относительно конфликта (B. R. Schlenker, T. V. Bonoma, 1978; A. Zornoza, P. Ripoll, 2002) $[23,25]$.

Для цели нашего исследования интерес представляют прежде всего опросники и то, для изучения каких аспектов конфликта они предназначены. Особое значение имеют инструменты, которые позволяют оценивать разные грани самой природы конфликта на разных уровнях его проявления в производственных группах.

Опросники конфликта в группах и командах. В настоящее время существует достаточно широкий спектр опросников, предназначенных для изучения конфликта в малых производственных группах. Их можно разделить на несколько категорий в зависимости от предмета измерения (во внимание не будут приниматься анкеты). Во-первых, значительная часть методик разработана для изучения межличностных конфликтов в группе. Причем одни из них направлены на оценку меры выраженности межличностного конфликта в общем, а другие - на диагностику типов межличностного конфликта. Так, Опросник межличностных конфликтов на работе включает пять пунктов, отражающих конфликт между членами команды относительно проблем, возникающих в процессе совместного выполнения работы [17]. Мера выраженности конфликта определяется степенью общей напряженности в группе, которая может быть связана как с индивидуальными ценностями членов команды, так и с особенностями выполнения ими работы. Шкала организационного конфликта состоит из пяти пунктов, в которых фокус делается на активной враждебности членов в группе [6]. В этих двух опросниках оценка испытуемыми пунктов осуществляется посредством пятибалльной шкалы типа шкалы Лайкерта. Модульная методика диагностики межличностного конфликта содержит шесть пунктов [1]. По каждому пункту члены группы оценивают друг друга по пятибалльной шкале (от + 5 до-5), содержание числовых значений которой различно в разных пунктах. Однако в этом опроснике нет ни одного айтема, который действительно бы отображал конфликт. Содержание пунктов связано либо с отношением (положительным/отрицательным) одних членов к другим, либо с их оценкой друг друга по некоторым характеристикам.

Другие опросники этой категории предназначены для измерения степени выраженности типов межличностного конфликта в производственных группах и командах. Наиболее широкое распространение за рубежом получила Шкала внутригруппового конфликта, которая имеет ряд модификаций по стимульному материалу (пунктам) и количеству субшкал. 
В частности, в двухфакторном варианте эта шкала позволяет изучать два типа межличностного конфликта-конфликт отношения и конфликт задачи [18], а в трехфакторной версии, реже используемой, - помимо этих двух типов еще и конфликт процесса [19]. В первом случае опросник включает восемь пунктов, а во втором - 14. В обоих случаях используется пятибалльная оценочная шкала типа шкалы Лайкерта. Надо сказать, что Шкала внутригруппового конфликта, несмотря на ее активное использование в научных исследованиях, не лишена недостатков: а) некоторые пункты вызывают вопросы с точки зрения содержательной валидности, например, когда речь идет только о разногласиях, что однозначно не свидетельствует о конфликте; б) в ряде версий опросник содержит одни пункты в виде вопросов, а другие - в форме утверждений, что делает его внутренне не унифицированным; в) некоторые пункты одних и тех же субшкал фактически одинаковы по форме и содержанию и, тем самым, дублируют друг друга; г) в каких-то модификациях пункты опросника сформулированы в прошедшем времени, так что посредством него изучают прошлые, а не актуальные в настоящее время конфликты и др. Для преодоления этих и других ограничений был разработан, проверен на надежность и валидность, Onросник типов межличностного конфликта в группе (А. В. Сидоренков и др., 2014) [5]. Он представлен в двух формах (для гражданских производственных групп и для воинских подразделений) и включает две субшкалы для изучения таких типов конфликта, как деятельностно-ориентированный и субъектноориентированный. Опросник содержит восемь пунктов в виде утверждений, по четыре в каждой субшкале. Оценка осуществляется посредством семибалльной шкалы. Одно из преимуществ построения этого опросника заключается в том, что он позволяет оценивать меру выраженности указанных типов конфликта как в группе в целом, так и внутри неформальных подгрупп в группе.

Во-вторых, некоторые опросники предназначены для изучения не столько выраженности самого межличностного конфликта в группе, сколько причин его возникновения. Например, Опросник прогнозирования межличностных производственных конфрликтов содержит пять пунктов, отражающих возможные причины конфликта [2]. По этим пунктам члены группы оценивают друг друга посредством трех вариантов ответа.

В-третьих, ряд опросников разработан для определения способов (стилей) поведения членов группы в конфликте или управления конфликтом. Так, Шкала стратегий поведения в конфликте направлена на оценку следующих стратегий: кооперация, конкуренция, поддержка и избегание [9]. Кроме того шкала позволяет установить, как выбранный стиль поведения влияет на урегулирование конфликтов в группе. Эта методика включает 
двадцать пунктов, которые оцениваются членами группы по пятибалльной шкале типа шкалы Лайкерта. Опросник стилей поведения в конфликте предназначен для изучения двух базовых стилей поведения (кооперативный и конкурентный) и один дополнительный (действенный, т. е. способность команды справиться с конфликтной ситуацией) [7]. Базовые шкалы включают в себя по пять пунктов, а дополнительная шкала-шесть айтемов. В этой методике используется семибалльная шкала Лайкерта. Опросник урегулирования конфликта на групповом и межгрупповом уровнях содержит четыре субшкалы: кооперативное урегулирование внутригруппового конфликта, конкурентное урегулирование внутригруппового конфликта, кооперативное урегулирование межгруппового конфликта и конкурентное урегулирование межгруппового конфликта [15]. Все субшкалы включают в себя по четыре пункта, за исключением субшкалы кооперативного урегулирования внутригруппового конфликта. Оценка осуществляется посредством семибалльной шкалы Лайкерта. Опросник организационного конфликта направлен на изучение стилей урегулирования межличностного конфликта: уступчивость, сотрудничество, доминирование, избегание и компромисс [22]. Он содержит двадцать восемь пунктов и представлен в трех формах, предназначенных для изучения поведения сотрудников в конфликте с руководителями, подчиненными и коллегами. Оценки по пунктам методики делаются с помощью семибалльной шкалы Лайкерта. Позднее опросник был модифицирован и дополнен двумя стратегиями поведения в конфликте (игнорирование и эмоции), так что в новой версии он включает сорок шесть пунктов [10]. Основным ограничением такого рода опросников является отсутствие единого подхода к классификации стратегий поведения в конфликте.

Итак, обзор существующих опросников показал, что одни из них направлены на изучение меры выраженности межличностного конфликта или его типов в производственных группах, другие-на выявление причин возникновения конфликтов в группе, а третьи-на оценку доминирующих у членов группы способов поведения в конфликте. Применительно к первой категории опросников надо отметить, что в любой группе могут возникать конфликты не только на межличностном уровне, но и на групповом и микрогрупповом уровнях. В последних случаях речь идет о конфликте отдельных работников с группой и неформальной подгруппой в группе. Нами не были обнаружены тест-опросники, которые предназначались бы для исследования этих уровней проявления конфликта, что явилось одной из причин создания соответствующего инструментария в рамках данной работы.

Цель исследования-разработка на определенной концептуальной основе опросника типов группового и микрогруппового конфликта в производственных группах. 
Задачи исследования: 1) создание опросника, позволяющего одновременно измерять меру выраженности группового и микрогруппового конфликта по двум его типам: деятельностно-ориентированному и субъектно-ориентированному; 2) обеспечение в процессе разработки опросника максимально возможного его соответствия (по трактовке конфликта и его типов, операциональным признакам конфликта, принципам построения, количеству и форме тестовых пунктов) ранее созданному опроснику типов межличностного конфликта в группе; 3) проверка опросника на надежность и валидность.

Создание такого инструментария и его дальнейшее применение в сочетании с опросником типов межличностного конфликта позволит получать полную картину об уровнях и типах проявления конфликта в производственных группах.

\section{Концептуальная платформа}

В основу разработки опросника были положены определение и модель конфликта в группе, представленные в микрогрупповой теории (А. В. Сидоренков, 2010; А. В. Сидоренков и др., 2014) [4, 5]. Конфликт понимается как форма дезинтегративного взаимоотношения и взаимодействия между субъектами (индивид, подгруппа, группа), порождаемая обострением противоречия и выражающаяся в активно-негативной направленности сторон по отношению друг к другу.

Многомерная модель конфликта содержит два измерения: уровни и типы конфликта. В малой группе существует пять возможных уровней проявления конфликта: 1) межличностный - конфликт между индивидами внутри подгруппы, между членами разных подгрупп, между «самостоятельными» членами группы, между представителями подгрупп и «самостоятельными» членами группы; 2) микрогрупповой - конфликт между индивидом и подгруппой; 3) групповой-конфликт между индивидом и группой; 4) межмикрогрупповой - конфликт между подгруппами как коллективными субъектами; 5) между подгруппой и группой-конфликт между подгруппой и группой.

Каждый уровень выражается в двух типах конфликта: деятельностноориентированном и субъектно-ориентированном. Деятельностно-ориентированный конфликт (ДОК) - конфликт, в котором «активно-негативная направленность сторон друг на друга связана с ключевыми аспектами совместной (профессиональной) работы», тогда как субъектно-ориентированный конфликт (СОК) предполагает «негативную активность сторон относительно друг друга в сфере коммуникации, которая прямым образом не связана с задачами и содержанием основной совместной работы» [5, с. 107].

Данная модель ранее успешно использовалась в создании опросника типов межличностного конфликта в группе. Ее применение для построения 
опросника типов группового и микрогруппового конфликта позволит в перспективе использовать одновременно оба опросника для комплексного изучения на единой теоретической основе, по крайней мере, трех уровней конфликта (по два типа на каждом из них) в производственных группах.

\section{Методика}

Опросник типов группового и микрогруппового конфликта (ОТГМК). С начала разработки опросника предполагалось, что он будет включать две субшкалы, соответствующие двум указанным типам конфликта (ДОК и СОК) на групповом и микрогрупповом уровнях его проявления. Стимульный материал создавался в виде утверждений, которые должны были отображать данное выше определение конфликта, понимание того или иного его типа, а также как минимум один из операциональных признаков конфликта: ссору, раздражительность и гнев, возмущение, упреки и критику, оскорбление и унижение, угрозу. Первоначально стимульный материал содержал 12 пунктов, по шесть в каждой субшкале, однако в результате оценки опросника было оставлено восемь пунктов, по четыре в каждой из двух субшкал. Оценка испытуемыми каждого пункта осуществляется по семибалльной шкале типа Лайкерта (от 1 - «полностью не согласен» до 7-«полностью согласен»). Таким образом, по природе изучаемых типов конфликта, количеству пунктов и шкале оценок этот опросник идентичен опроснику типов межличностного конфликта в группе. Такая унификация двух методик создает предпосылку для комплексного исследования разных уровней конфликта, сравнения меры их выраженности, связей и соразмерностей между ними.

В окончательном варианте опросник включает две части: 1) «Группа в целом» (для оценки типов группового конфликта); 2) «Общность тех, с кем поддерживаю тесные отношения» (для изучения типов микрогруппового конфликта). Инструкция и фрагмент опросника 2 представлены в таблице 1. Для решения научных и практических задач методика может применяться в полной форме или в одной из двух частей. Если интерес представляет групповой и/или микрогрупповой конфликт в контексте всей группы, то обследование может проводиться в анонимной форме. Когда необходимо исследовать проявление того или иного уровня конфликта в неформальных подгруппах, то дополнительно испытуемым дается опросник 1, представленный в таблице 1, для выявления неформальных подгрупп в группе.

Обработка результатов обследования проходит, как минимум, в два этапа. Первый этап предполагает подсчет индивидуальных показателей путем суммирования оценок каждого респондента по каждой субшкале: ДОК (пункты №№ 1, 2, 3, 4) и СОК (пункты №№ 5, 6, 7, 8). Индивидуальные показатели рассчитываются отдельно для первой и для второй частей 
опросника. На втором этапе рассчитываются коэффициенты: а) группового конфликта ДОК-Г и/или СОК-Г, определяемые как среднее арифметическое индивидуальных показателей всех членов группы, участвовавших в обследовании (по части 1); б) микрогруппового конфликта ДОК-МГ и/или СОК-МГ, определяемые как среднее арифметическое индивидуальных показателей всех членов группы, участвовавших в обследовании (по части 2). Если необходимо изучить один или оба уровня конфликта в неформальных подгруппах, то, после выделения в группе подгрупп и определения их состава, для каждой из них рассчитываются ДОК-Г-П и/или СОК-Г-П, ДОК-МГ-П и/или СОК-МГ-П. Алгоритм расчета такой же, как описан выше, за исключением того, что расчеты проводятся по показателям членов каждой подгруппы в отдельности. Значение каждого коэффициента может принимать значения от 4 до 28, при этом высокие показатели свидетельствуют о сильной выраженности группового/микрогруппового конфликта, а низкие-о слабой.

Таблица 1.

Инструкция и фрагмент стимульного материала ОТГМК

\begin{tabular}{|c|c|c|}
\hline \multicolumn{3}{|c|}{ ОПРОСНИК 1} \\
\hline \multicolumn{3}{|c|}{$\begin{array}{c}\text { Выберите из Вашей группы (управления, отдела, смены...) тех людей, с которыми } \\
\text { Вы поддерживаете наиболее тесные отношения. (Количество таких людей не обя- } \\
\text { зательно должно соответствовать количеству граф.) }\end{array}$} \\
\hline \multicolumn{2}{|l|}{1} & 3 \\
\hline 2 & \multicolumn{2}{|l|}{4} \\
\hline \multicolumn{3}{|c|}{ ОПРОСНИК 2} \\
\hline \multicolumn{3}{|c|}{$\begin{array}{l}\text { Инструкция: «Прочитайте утверждения и оцените их относительно: 1) Вашей } \\
\text { группы: управления, отдела, бригады, смены и т. д. (с левой стороны утверж- } \\
\text { дений); 2) общности тех, с кем Вы поддерживаете наиболее тесные отноше- } \\
\text { ния в Вашей группе (с правой стороны утверждений). Оценка производится } \\
\text { по семибалльной шкале, где «1» означает «полностью не согласен», «7»-«полно- } \\
\text { стью согласен», «4»- «нечто среднее»; остальные баллы выражают разную про- } \\
\text { межуточную меру Вашего согласия/несогласия. По каждому пункту допускается } \\
\text { выбор только одного числового значения. Не оставляйте задания без ответов». }\end{array}$} \\
\hline Группа в целом & Утверждения & $\begin{array}{c}\text { Общность тех, } \\
\text { с кем поддерживаю } \\
\text { тесные отношения }\end{array}$ \\
\hline & $\begin{array}{l}\text { 1. Нередко вызывает раздражение у от- } \\
\text { дельных членов, игнорируя их предло- } \\
\text { жения (мнения) по рабочим вопросам. }\end{array}$ & \\
\hline & $\begin{array}{c}\text { 2. Зачастую навязывает некоторым } \\
\text { работникам свои решения по произ- } \\
\text { водственным вопросам, вызывая у них } \\
\text { возмущение, сопротивление. }\end{array}$ & \\
\hline
\end{tabular}


Участники исследования. В исследовании приняли участие 18 производственных групп, представляющих собой первичные структурные подразделения в организациях разного профиля деятельности (газовая отрасль, военная служба, туристический сервис, торговля и др.) или полные коллективы малочисленных компаний. Все группы были организационно сформированы, срок жизнедеятельности каждой группы к моменту исследования составлял не менее 1 года. Численность каждой группы варьировалась от 4 до 20 человек ( $M$ = 11.1). Общее количество респондентов составило 200 человек. Выборка включала в себя 65 \% мужчин и $35 \%$ женщин, возраст испытуемых составлял от 24 до 52 лет (M = 37.4).

Процедура оценки опросника. Для оценки содержательной валидности опросника были привлечены три специалиста-психолога с большим стажем профессиональной деятельности, специализирующиеся в области социальной психологии. По предоставленной спецификации ими оценивалось соответствие каждого пункта определенному типу конфликта по пятибалльной шкале (от 1 - «полностью не соответствует» до 5- «полностью coответствует»). В качестве экспертов для оценки очевидной валидности были привлечены 25 работников, отобранных случайным образом из обследованных групп. Им предлагалось оценить по пятибалльной шкале (от 1 - «совершенно непонятно» до 5-«полностью понятно»), насколько понятен по смыслу каждый из пунктов опросника.

Статистическая обработка данных проводилась с использованием программного продукта SPSS17.0. Осуществлялся расчет описательных статистик (средних значений и стандартных отклонений) и коэффициента а Кронбаха, выполнялась оценка эмпирического распределения по критерию Колмогорова-Смирнова, проводился факторный анализ с использованием метода главных компонент и вращения Варимакс с нормализацией Кайзера (использовались показатели структурной матрицы).

\section{Результаты и их обсуждение}

Показатели содержательной валидности пунктов опросника, рассчитанные как средние значения соответствующих экспертных оценок, варьировали от 4.66 до 5.0, а очевидной валидности - от 4.46 до 5.0 (таблица 2). Как видно, все пункты опросника можно считать обладающими данными видами валидности.

В целях соблюдения оптимального объема статьи и упрощения процедуры анализа, во внимание будут приниматься только результаты по разделу опросника «Группа в целом». Проведенный факторный анализ данных исследования позволил оценить дискриминантную валидность разработанной методики (таблица 2). По результатам анализа из стимульного материала были исключены пункты с низкими значениями показателей факторных 
нагрузок. Двухфакторный анализ итогового варианта опросника показал, что факторные нагрузки пунктов, соответствующих субшкале ДОК, варьируются в пределах от .801 до .927, а соответствующих субшкале СОК - от .920 до .954. При этом собственное значение фактора F1 (субшкала ДОК) составило 1.19, дисперсия - 14.83\%, а фактора F2 (субшкала СОК) - 5.88 с дисперсией 73.49\%. Таким образом, в соответствии с критерием Кайзера, выделение двух факторов является необходимым и достаточным для данной методики, в связи с чем можно с уверенностью утверждать об обоснованности двух субшкал ОТГМК.

Таблича 2.

Показатели валидности и факторного анализа ОТГМК

\begin{tabular}{|c|c|c|c|c|c|c|}
\hline \multirow[t]{2}{*}{ Пункты } & \multicolumn{3}{|c|}{$\begin{array}{c}\text { Деятельностно- } \\
\text { ориентированный } \\
\text { конфликт }\end{array}$} & \multicolumn{3}{|c|}{$\begin{array}{c}\text { Субъектно- } \\
\text { ориентированный } \\
\text { конфликт }\end{array}$} \\
\hline & CB & OB & $\begin{array}{c}\Phi H \\
\text { (F1) }\end{array}$ & CB & OB & $\begin{array}{l}\text { ФH } \\
\text { (F2) }\end{array}$ \\
\hline $\begin{array}{l}\text { 1. Нередко вызывает раздражение у от- } \\
\text { дельных членов, игнорируя их пред- } \\
\text { ложения (мнения) по рабочим вопросам }\end{array}$ & 4.66 & 4.46 & .873 & - & - & .326 \\
\hline $\begin{array}{c}\text { 2. Зачастую навязывает некоторым } \\
\text { работникам свои решения по произ- } \\
\text { водственным вопросам, вызывая у них } \\
\text { возмущение, сопротивление }\end{array}$ & 5 & 4.73 & .927 & - & - & .689 \\
\hline $\begin{array}{c}\text { 3. Часто резко критикует кого-то из сво- } \\
\text { их коллег за то, как они выполняют про- } \\
\text { фессиональные обязанности }\end{array}$ & 5 & 4.73 & .894 & - & - & .775 \\
\hline $\begin{array}{c}\text { 4. Нередко обменивается с отдель- } \\
\text { ными членами взаимными упреками } \\
\text { и претензиями в процессе выполнения } \\
\text { работы }\end{array}$ & 5 & 4.8 & .801 & - & - & .667 \\
\hline $\begin{array}{l}\text { 5. Зачастую недружелюбно относится } \\
\text { к некоторым членам, даже если они ис- } \\
\text { правно выполняют свои обязанности }\end{array}$ & - & - & .643 & 5 & 5 & .954 \\
\hline $\begin{array}{c}\text { 6. Нередко открыто и резко высказы- } \\
\text { вается относительно личности кого-то } \\
\text { из работников }\end{array}$ & - & - & .540 & 5 & 5 & .920 \\
\hline $\begin{array}{c}\text { 7. Часто нелицеприятно высказывается } \\
\text { в адрес отдельных членов по поводу их } \\
\text { привычек, манеры поведения }\end{array}$ & - & - & .548 & 5 & 4.93 & .950 \\
\hline $\begin{array}{c}\text { 8. Нередко навязывает некоторым чле- } \\
\text { нам мнение по вопросам, не связанным } \\
\text { с работой, вызывая у них раздражение } \\
\text { и возмущение }\end{array}$ & - & - & .445 & 5 & 4.8 & .951 \\
\hline
\end{tabular}

Примечание: 1. В табличе приведены пункты, которые остались в окончательной версии шкалы. 2. CB-содержательная валидность (среднее значение), ОВ-очевидная валидность (среднее значение), ФН-факторная нагрузка, F-фактор. 
Корреляция между двумя факторами, соответствующими субшкалам ДОК и СОК, оказалась равной .63, что указывает на наличие средне выраженной положительной взаимосвязи между анализируемыми типами конфликта. Такая связь объясняется тем, что указанные субшкалы измеряют единый феномен групповой активности - конфликт, -хотя и по отличающимся аспектам. В целом, результаты проведенного факторного анализа свидетельствуют о достаточной дискриминантной валидности разработанного опросника.

Коэффициенты а Кронбаха рассчитывались отдельно для каждой из субшкал методики. Для субшкалы ДОК $a=.915$, для субшкалы СОК $a=.962$, что позволяет судить о высокой надежности-согласованности ОТГМК.

Расчет средних значений и стандартных отклонений, а также расчет статистик критерия D Колмогорова-Смирнова для субшкал ДОК и COK показал, что эмпирическое распределение приближается к нормальному (таблица 3). Из этого можно заключить, что обе субшкалы обладают достаточной диагностической силой. Кроме того, указанные описательные статистики могут использоваться в практических исследованиях в качестве основы выделения статистических зон для оценки меры выраженности того или иного типа группового и микрогруппового конфликта в конкретных производственных группах.

Таблица 3.

Средние значения, стандартные отклонения и статистики критерия D Колмогорова - Смирнова для субшкал ОТГМК

\begin{tabular}{|c|c|c|c|}
\hline Субшкалы ОтгмК & Среднее значение & $\begin{array}{c}\text { Статистика } \\
\text { Стандартное } \\
\text { отклонение }\end{array}$ & $\begin{array}{c}\text { критерия D } \\
\text { Колмогорова- } \\
\text { Смирнова }\end{array}$ \\
\hline ДОК & 14.48 & 3.10 & $.651^{*}$ \\
\hline СОК & 11.29 & 3.37 & $.534^{*}$ \\
\hline
\end{tabular}

Примечание: 1. $N=200$ членов малых прочзводственных групn. $2 .{ }^{*}-p \leq 0.01$.

\section{Выводы}

Для получения полной картины проявления конфликтов в производственных группах необходимо принимать во внимание разные уровни и типы конфликта. Для их изучения актуально создание соответствующего инструментария. Разработанный Опросник типов группового и микрогруппового конфликта обладает достаточной валидностью и надежностью по всем проанализированным показателям. Обоснованность выделения двух субшкал методики, соответствующих двум типам конфликта, оказалась подтверждена результатами эмпирического исследования. 
Указанная методика может применяться для решения задач как научноисследовательского, так и прикладного характера. Вместе с разработанным ранее Опросником типов межличностного конфликта в группе она составляет методический комплекс, позволяющий изучать конфликт на всех уровнях его проявления (межличностном, микрогрупповом и групповом) по каждому типу (деятельностно-ориентированному и субъектно-ориентированному), как по отдельности, так и во взаимосвязи. Это открывает широкие перспективы дальнейшего исследования феномена конфликта в контексте связей и соразмерности его уровней и типов, его связей с разными групповыми явлениями и процессами, в том числе с групповой эффективностью.

\section{Литература}

1. Анцупов А. Я., Шипилов А. И. Конфликтология. - М.: ЮНИТИ, 1999.

2. Лебедев А. Н. Прогнозирование и профилактика межличностных производственных конфликтов в условиях нововведений // Психологический журнал. - 1992. - № 6. - С. 71-79.

3. Макринова Е. И., Пахомова Ю. А. Диагностика противоречий и конфликтов в социально-трудовой сфере организаций потребительской кооперации // Вестник Белгородского университета кооперации, экономики и права. - 2010. - № 3. - С. 30-35.

4. Сидоренков А. В. Малая группа и неформальные подгруппы: микрогрупповая теория. - Ростов н/Д: Изд-во ЮФУ, 2010.

5. Сидоренков А. В., Локтева Е. А., Мкртчян А. А. Инструментарий исследования типов межличностных противоречий и конфликтов в малых группах // Психологический журнал. - 2014. - Т. 35. - № 2. - С. 103-117.

6. Afzal H., Khan M. A., Ali l. Linkage between Employee's Performance and Relationship Conflict in Banking Scenario // International Journal of Business and Management. - 2009. - no. 4 (7). - pp. 19-25.

7. Alper S., Tjosvold D., Law K. S. Conflict management, efficacy, and performance in organizational teams // Personnel Psychology. - 2000. - no. 53 (3). pp. 625-642.

8. Bales R. F., Cohen S. P. SYMLOG. A system for the multiple level observation of groups. - N. Y.: Free Press, 1979.

9. Barker J., Tjosvold D., Andrews I. R. Conflict Approaches of Effective and Ineffective Project Managers: A Field Study in a Matrix Organization // Journal of Management Studies. - 1988. - no. 25 (2). - pp. 167-178.

10. Behrendt H., Ben-Ari R. The Positive Side of Negative Emotion: The Role of Guilt and Shame in Coping with Interpersonal Conflict // Journal of Conflict Resolution. - 2012. - no. 56 (6). - pp. 1116-1138. 
11. Becker-Beck U. Soziale interaktion in gruppen: Struktur und prozeßanalyse // Social interaction in groups: Analyses of structure and process. Germany: Westdeutscher Verlag, 1997.

12. Boz M., Martínez I., Munduate L. Rompiendo las Consecuencias Negativas del Conflicto de Relación en el Trabajo: El Rol Moderador del Enriquecimiento Trabajo-Familia y el Apoyo del Supervisor // Revista de Psicología del Trabajo y de las Organizaciones. - 2009. -V. 25. - no. 2. - pp. 113-121.

13. Fang L., Hipel K. W., Kilgour D. M., Peng X. The Decision Support System GMCR II // Group decision and negotiation. - 2001. -V. 10. - no. 2. - pp. 159-175.

14. Farmer S. M., Roth J. Conflict-Handling behavior in work groups: effects of group structure, decision processes and time // Small Group Research. 1998. - V. 29. - no. 6. - pp. 669-713.

15. Hempel P. S., Zhang Zh., Tjosvold D. Conflict management between and within teams for trusting relationships and performance in China // Journal of Organizational Behavior. - 2009. - no. 30. - pp. 41-65.

16. Hipel K. W., Kilgour D. M., Fang L., Peng X. The Decision Support System GMCR II in negotiations over Groundwater Contamination // Proceedings IEEE SMC International Conference. -Tokyo, Japan, 1999. - pp. 942-948.

17. Ilies R., Johnson M. D., Judge T. A., Keeney J. A within-individual study of interpersonal conflict as a work stressor: dispositional and situational moderators // Journal of organizational behavior. - 2011. -V. 32. - no. 1. pp. 44-64.

18. Jehn K. A. A qualitative analysis of conflict types and dimensions in organizational groups // Administrative Science Quarterly. - 1997. -V. 42. - pp. 530-557.

19. Jehn K. A., Mannix E. A. The dynamic nature of conflict: A longitudinal study of intragroup conflict and group performance // Academy of Management Journal. - 2000. - no. 44. - pp. 238-251.

20. Kilgour D. M., Fang L., Hipel K. W. Negotiation Support Using the Decision Support System GMCR // Group Decision and Negotiation. - 1996. - no. 5. pp. 371-384.

21. Poole M. S. Decision development in small groups II: a study of multiple sequences in decision making // Communication monographs. - 1983. no. 50 (3). - pp. 206-232.

22. Rahim M. A., Magner N. R. Confirmatory factor analysis of the styles of handling interpersonal conflict: first-order factor model and its invariance across groups // Journal of applied psychology. - 1995. -V. 80 (1).

23. Schlenker B. R., Bonoma T. V. Fun and games: the validity of games for the study of conflict // Journal of conflict resolution. - 1978. - no. 22 (1). - pp. 7-38.

24. Wall V. D., Nolan L. L. Small group conflict: A Look at Equity, Satisfaction and Styles ofConflict Management //Small group behavior. - 1987. - no. 18. -pp. 188-211. 
25. Zornoza A., Ripoll P., Peiry J. M. Conflict management in groups that work in two different communication contexts: Face-To-Face and Computer-Mediated Communication // Small group research. - 2002. -V.33. - no. 5. - pp. 481-508.

\section{References}

1. Antsupov A. Ia., Shipilov A. I. Konfliktologiia [Conflictology]. Moscow, IuNITI Publ., 1999.

2. Lebedev A. N. Prognozirovanie i profilaktika mezhlichnostnykh proizvodstvennykh konfliktov v usloviiakh novovvedenii [Prediction and prevention of interpersonal conflicts in groups of employees in the conditions of innovation]. Psikhologicheskii zhurnal - Psychological Journal, 1992, no. 6, pp. 71-79.

3. Makrinova E. I., Pakhomova lu. A. Diagnostika protivorechii i konfliktov v sotsial'no-trudovoi sfere organizatsii potrebitel'skoi kooperatsii [Diagnostics of contradictions and conflicts in the socio-labor sphere in organizations of consumer cooperation]. Vestnik Belgorodskogo universiteta kooperatsii, ekonomiki i prava - Vestnik of Belgorod University of Cooperation, Economics, and Law, 2010, no. 3, pp. 30-35.

4. Sidorenkov A. V., Lokteva E. A., Mkrtchian A. A. Instrumentarii issledovaniia tipov mezhlichnostnykh protivorechii i konfliktov $v$ malykh gruppakh [A small group and informal subgroups: microgroup theory]. Rostov-on-Don, Southern Federal University Publ., 2010.

5. Sidorenkov A. V., Lokteva E. A., Mkrtchian A. A. Instrumentarii issledovaniia tipov mezhlichnostnykh protivorechii i konfliktov v malykh gruppakh [Tools for studying types of interpersonal conflicts in small groups]. Psikhologicheskii zhurnal - Psychological Journal, 2014, V. 35, no. 2, pp. 103-117.

6. Afzal H., Khan M. A., Ali I. Linkage between Employee's Performance and Relationship Conflict in Banking Scenario. International Journal of Business and Management, 2009, no. 4 (7), pp. 19-25.

7. Alper S., Tjosvold D., Law K. S. Conflict management, efficacy, and performance in organizational teams. Personnel Psychology, 2000, no. 53 (3), pp. 625-642.

8. Bales R. F., Cohen S. P. SYMLOG. A system for the multiple level observation of groups. N. Y.: Free Press, 1979.

9. Barker J., Tjosvold D., Andrews I. R. Conflict Approaches of Effective and Ineffective Project Managers: A Field Study in a Matrix Organization. Journal of Management Studies, 1988, no. 25 (2), pp. 167-178.

10. Behrendt H., Ben-Ari R. The Positive Side of Negative Emotion: The Role of Guilt and Shame in Coping with Interpersonal Conflict. Journal of Conflict Resolution, 2012, no. 56 (6), pp. 1116-1138. 
11. Becker-Beck U. Soziale interaktion in gruppen: Struktur und prozeßanalyse: Social interaction in groups: Analyses of structure and process. Germany: Westdeutscher Verlag, 1997.

12. Boz M., Martínez I., Munduate L. Rompiendo las Consecuencias Negativas del Conflicto de Relación en el Trabajo: El Rol Moderador del Enriquecimiento Trabajo-Familia y el Apoyo del Supervisor. Revista de Psicología del Trabajo y de las Organizaciones, 2009, V. 25, no. 2, pp. 113-121.

13. Fang L., Hipel K. W., Kilgour D. M., Peng X. The Decision Support System GMCR II. Group Decision and Negotiation, 2001, V. 10, no. 2, pp. 159-175.

14. Farmer S. M., Roth J. Conflict-Handling behavior in work groups: effects of group structure, decision processes and time. Small Group Research, 1998, V. 29, no. 6, pp. 669-713.

15. Hempel P. S., Zhang Zh, Tjosvold D. Conflict management between and within teams for trusting relationships and performance in China. Journal of Organizational Behavior, 2009, no. 30, pp. 41-65.

16. Hipel K. W., Kilgour D. M., Fang L., Peng X. The Decision Support System GMCR II in negotiations over Groundwater Contamination. Proceedings IEEE SMC International Conference, Tokyo, Japan, 1999, pp. 942-948.

17. Ilies R., Johnson M. D., Judge T. A., Keeney J. A within-individual study of interpersonal conflict as a work stressor: dispositional and situational moderators. Journal of Organizational Behavior, 2011, V. 32, no. 1, pp. 44-64.

18. Jehn K. A. A qualitative analysis of conflict types and dimensions in organizational groups. Administrative Science Quarterly, 1997, V. 42, pp. 530-557.

19. Jehn K. A., Mannix E. A. The dynamic nature of conflict: A longitudinal study of intragroup conflict and group performance. Academy of Management Journal, 2000, no. 44, pp. 238-251.

20. Kilgour D. M., Fang L., Hipel K. W. Negotiation Support Using the Decision Support System GMCR. Group Decision and Negotiation, 1996, no. 5, pp. 371-384.

21. Poole M. S. Decision development in small groups II: a study of multiple sequences in decision making. Communication Monographs, 1983, no. 50 (3), pp. 206-232.

22. Rahim M. A., Magner N. R. Confirmatory factor analysis of the styles of handling interpersonal conflict: first-order factor model and its invariance across groups. Journal of Applied Psychology, 1995, V. 80 (1).

23. Schlenker B. R., Bonoma T. V. Fun and games: the validity of games for the study of conflict. Journal of Conflict Resolution, 1978, no. 22 (1), pp. 7-38.

24. Wall V. D., Nolan L. L. Small group conflict: A Look at Equity, Satisfaction 
RUSSIAN PSYCHOLOGICAL JOURNAL・ 2016 VOL. 13 \# 3

and Styles of Conflict Management. Small Group Behavior, 1987, no. 18, pp. 188-211.

25. Zornoza A., Ripoll P., Peiry J. M. Conflict management in groups that work in two different communication contexts: Face-To-Face and ComputerMediated Communication. Small Group Research, 2002, V. 33, no. 5, pp. 481-508. 